\title{
Emergency evacuation simulation in public facilities based on CTM
}

\author{
Zhou He-Cheng, Zhao Xiao-Fang \\ Beijing Key Lab of Urban Intelligent Traffic Control Technology, North China University of \\ Technology, Beijing, 100041 \\ $575542221 @ q q . c o m$
}

Keywords: Cell transmission model, Repulsive force, Evacuation efficiency

\begin{abstract}
Based on cell transmission model, this paper formulates a dynamic simulation model for studying emergency evacuation in a public facility to improve evacuation efficiency, in terms of inefficiency of static simulation model. Considering evacuation population, evacuation rate, the size of buildings, diffusion probability of disaster, repulsive force between evacuees and other parameters, this paper first calculates the evacuation efficiency and evacuation time, and then proposes a evacuation plan. The simulation results show that the dynamic simulation model is advantageous over the static and the appropriate repulsive parameters can improve the evacuation efficiency.
\end{abstract}

\section{Introduction}

Personnel emergency evacuation is one of the hotspot in research of security in the world, generally having two kinds of static and dynamic simulation model. Real-time simulation of static model is not accurate and objective enough to simulate the change of information in the simulation area, so this paper uses a dynamic simulation model. Compared with the simulation results of static, we put forward feasible suggestions. Based on the theory of complex evacuation and conventional modeling method, and on the basis of CTM and multi-agent technology for evacuation modeling practice, we put forward time function for the process of evacuation [1], and established a dynamic simulation model for evacuation plan, which calculated optimal path by dynamic, thus providing evacuation model of dynamic evacuation plan, at the same time, according to the scheme for dynamic simulation. Through the main body of individual behavior of the evacuation model, observe the superiority of the dynamic model. With the analysis of the simulation data, repulsion between evacuees, disaster diffusion probability, and the influence of size on the emergency evacuation area to evacuation efficiency, it is concluded that the dynamic relationship between them.

\section{The establishment of the evacuation zone mode}

environment modeling

This paper is based on NO.5 Screen Wanda International cinema located in ShiJingshan, Beijing, where lays 160 seats, covering 20*20 square meters. In the middle of the screen, there have 2 evacuation passageways. And there is a big and a small emergency exit in the front of it. In the paper, we have ignored the influence of seats while evacuating.

Evacuation model in this paper will simulate the building entity in public, which is an $x^{*} \mathrm{y}$ two-dimensional space abstract. Everyone covers a space of $0.5 \mathrm{~m}$ gridding, thus the whole 
two-dimensional space will be divided into an area made of $0.5^{*} 0.5$ gridding, each gridding concluding 2 states, covered and uncovered.

CTM mode

\subsubsection{Introduction of CTM}

Make single staff as the smallest unit, and set the length of each CTM as 0.5m.Thus, each evacuation staff is a CTM. For example, a single staff called a, chooses a gridding as his her next location from 8seats next to himlher. We assume a as $\mathrm{L}=(\mathrm{i}, \mathrm{j})$, then 8 grids next to him $\mathrm{j}-1),(\mathrm{i}-1, \mathrm{j}),(\mathrm{i}+1, \mathrm{j}),(\mathrm{i}+1, \mathrm{j}-1),(\mathrm{i}-1, \mathrm{j}-1),(\mathrm{i}-1, \mathrm{j}+1),(\mathrm{i}+1, \mathrm{i}+1),(\mathrm{i}, \mathrm{j}+1)$ respectively.

\subsection{2 basic behaviors for evacuation}

There are three most common behaviors: first, people who are familiar with the environment, knowing a lot about each evacuation route, will choose the quick route they think for safe-zone when in danger; second, people who are unfamiliar with the environment, choosing a route with a crowd, follow them to safe-zone. Third, people who are unfamiliar with the environment, choose the route by which they entered.

Behavior chosen by self

When evacuation staffs choose the quick route they think, the coordinate probability is ${ }^{[2]}$ :

$$
\begin{aligned}
& P_{(i, j)}=a(i, j) \times P_{D I S(i, j)} \\
& P_{D I S(i, j)}=\frac{\left[\max \left(D_{(i, j)}\right)-D_{(i, j)}\right]}{\left[\max \left(D_{(i, j)}\right)-\min \left(D_{(i, j)}\right)\right]}
\end{aligned}
$$

Behavior following crowd

When evacuation staffs choose the route with a crowd, the coordinate probability is:

$$
P_{D I R(i, j)}=\frac{N_{D I R(k)}}{\sum_{k=1}^{8} N_{D I R(\mathrm{k})}}
$$

\section{Behavior chosen enter route}

Network attractive probability that evacuation staff choose the same route by which they entered is in accordance with the first choice when they entered. When we are calculating the distance between the grid $M(i, j)$ to the evacuation exit $N(i, j)$, we set the evacuation exit as the enter position CTM chooses.

\subsubsection{Evacuee's mobile rules or routing selection}

Target gridding includes four states in evacuation: (1) free without competitor (2) free with competitor (3) covered by people (4) obstacles or walls. The above of things are some situations during the process of moving.

Rules for evacuation: (1) evacuation staff choose the most probable gridding coordinate as the next target coordinate. (2) Every coordinate can only hold one person in each time step, so each evacuation staff will judge whether the target coordinate is free before moving to next target coordinate. If there are more than 20 people choose the same gridding as the target gridding, they have to compete with their ability. The strong will enter the target coordinate, while others choose sub-optimal gridding in the area next to them. (3) Evacuation mode the staff chooses is related with the situation and the state, both physical and psychological, at that time. Of course, the behavior mode is not always the same. If evacuation staff chooses the same route by which they entered at first, they will automatically choose the exit with a few people when they suddenly find that exit in their sights. 


\section{The design of evacuation simulation system}

\subsection{The default conditions before the simulation}

(1) We choose one from three basic behaviors randomly for every evacuation staff to do evacuation; (2) evacuation starts once the simulation system works; (3) screen safety evacuation exits: 2; (4) evacuation staff:160; (5) the walls of screen: the four sides of the simulation interface;(6) every evacuation staff's speed is the same and remains unchanged; (7) every evacuee's physical condition is the same, assumed not any accidents before arriving at the exit.

2.2 Dynamic evacuation plan

2.2.1 the best evacuation route calculation in dynamic allocation[3]:

$$
\begin{aligned}
& \operatorname{avg} X=\sum_{i=1}^{n} X_{i} / n \\
& \operatorname{avg} Y=\sum_{i=1}^{n} Y_{i} / n \\
& L=\sqrt{(x-\operatorname{avg} X)^{2}+(y-\operatorname{avg} Y)^{2}} \\
& T=\frac{N}{P \times V}
\end{aligned}
$$

$\mathrm{X}$ : the horizontal axis of exit; $\mathrm{y}$ : the vertical axis of exit; $\mathrm{T}$ : cycle of simulation; $\mathrm{N}$ : the total number of simulation (160); P: successful number of evacuation in the circle of simulation; L: average distance from all CTM to the exits in the region, that is overall evacuation cost.

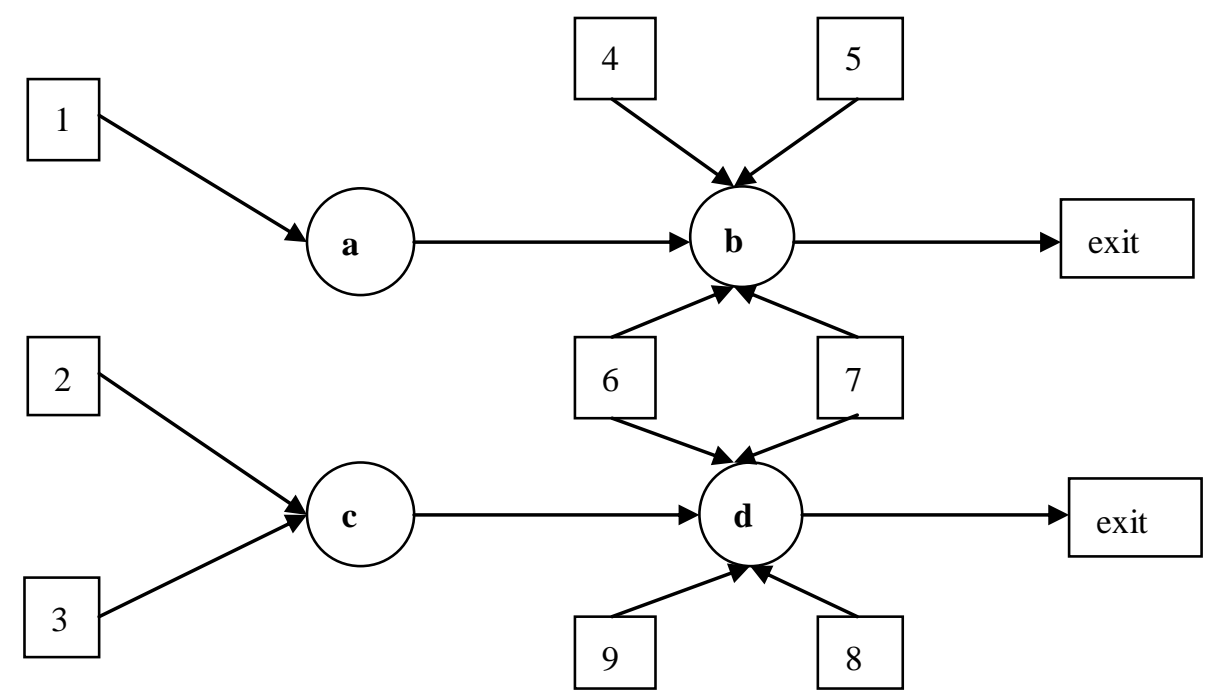

In the picture one, 1-9 represent the area of seats needed an evacuation, a-b, c-d are the two evacuation passageways of cinema.

\subsubsection{Introduction of dynamic evacuation plan}

In the picture, we can see the whole plan is divided into 2 areas of routes and 9 areas of seats. Each area of seats is a small unit of evacuation area. We can calculate evacuation time of every area with formula (7). During the process of evacuation simulation system, evacuation time will be calculated in every 5 circles. Dynamic evacuation plan is the value of $\mathrm{T}$ of two neighboring evacuation area. The value of $\mathrm{T}$ is bigger if the evacuation time is longer. The system will guide evacuation staffs evacuate from big $\mathrm{T}$ to small $\mathrm{T}$ according to the dynamic condition of $\mathrm{T}$, which improves evacuation efficiency of the whole building. 


\section{Analysis of Simulation Results}

In this paper, we use matlab as the simulation software, results including static and dynamic states. From table 2 to table 6 , the black frame represents wall of screen, yellow grids indicating evacuation signals of screen in the cinema, green rectangles being 2 safety exits of screen, blue circles being evacuation staff, and small red squares being diffusible hazard. Disaster diffusion probability is 0.04 , size of disaster damage is 3 , and staff's repulsion is 2 .

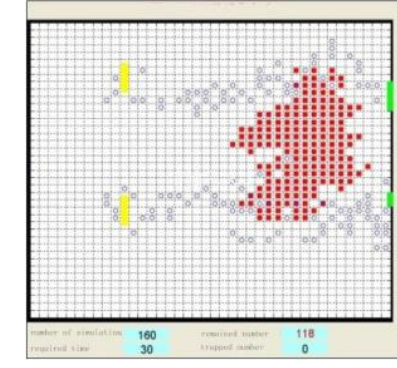

Picture2 30 circles status of the dynamic evacuation plan

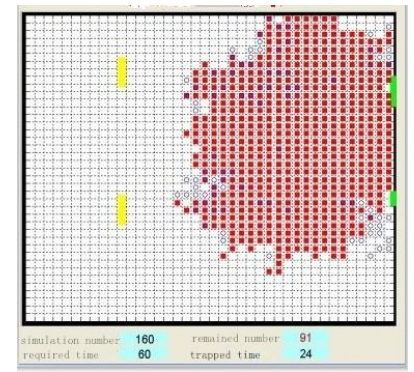

Picture 360 circles status of the dynamic evacuation plan

Table 1 the evacuation rate and average evacuation

\begin{tabular}{ccccc}
\multicolumn{5}{c}{ rate } \\
\hline PLAN & $\begin{array}{c}\text { Evacu- } \\
\text { ees }\end{array}$ & $\begin{array}{c}\text { Evac- } \\
\text { ueed } \\
\text { people }\end{array}$ & $\begin{array}{c}\text { Evacua- } \\
\text { tion rate } \\
(\%)\end{array}$ & $\begin{array}{c}\text { Average } \\
\text { evacuati- } \\
\text { on rate }\end{array}$ \\
\hline $\begin{array}{c}\text { No-evacuat } \\
\text { ion } \\
\text { Dynamic } \\
\text { evacuation } \\
\text { Static }\end{array}$ & 160 & 92 & $59.1 \%$ & 2.49 \\
\hline
\end{tabular}

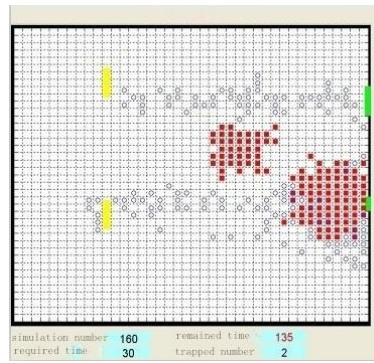

Picture 430 circles status of the static evacuation plan

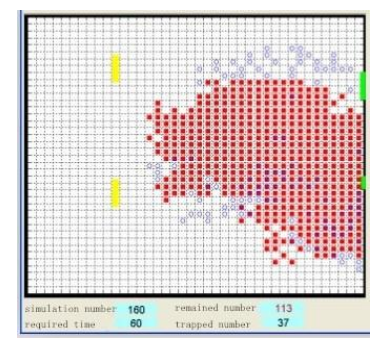

Picture 560 circles status of the static evacuation plan

Table 2 the repulsive force and evacuation

\begin{tabular}{ccccc} 
efficiency \\
\hline Repulsive force & 1 & 2 & 4 & 6 \\
\hline Evacuees & 107 & 134 & 121 & 100 \\
Evacuation time & 45 & 30 & 57 & 70 \\
Average rate & $66 \%$ & $83 \%$ & $75 \%$ & $62.5 \%$ \\
\hline
\end{tabular}

Table 3 disaster hazard level and evacuation efficiency

\begin{tabular}{ccccc}
\hline $\begin{array}{c}\text { Disaster hazard } \\
\text { level }\end{array}$ & 1 & 3 & 5 & 7 \\
\hline evacuees & 160 & 134 & 90 & 68 \\
Evacuation time & 15 & 30 & 54 & 41 \\
Average rate & $100 \%$ & $83 \%$ & $56.2 \%$ & $42.5 \%$ \\
\hline
\end{tabular}

In table 1, it shows the average of ten simulations. Comparing 60 circles of dynamic evacuation plan and static evacuation plan, we found that dynamic evacuation plan can guide evacuation staff to move from area with high risk and long evacuation time to area with low risk and short evacuation circle, so as to evacuate more people within the same amount of time. From simulations we can also found that, the evacuation efficiency of dynamic plan is much higher than that of static plan. When disaster diffusion probability parameter is below 0.04 , the number of stranded people is 
found about 0 . While, when the parameter is too high, the number will be excessive. Then there is nothing meaningful of simulations. So in this paper, we choose 0.04 as disaster diffusion probability parameter. In addition, as shown in table 2, we found that the relationship between evacuation efficiency and repelling force of personnel is related with time of simulation. The evacuation efficiency decreases after the first increases with the increase of repelling force. When disasters occur shortly in the movie theater, the density of personnel is low in evacuation path, and evacuation efficiency is gradually increasing. But with the rise of density of stream of people, repulsive force between personnel increases, and the evacuation efficiency decreases, consistent with the actual. When harm value is small, there can be $100 \%$ evacuation. As the level of disaster increases, the evacuation rate gradually reduces, consistent with actual also, as shown in table 3 .

\section{Conclusions}

From the simulation results of the screen, we can see the practicability and necessity of dynamic evacuation plan. Under the premise of bad effect of static evacuation, dynamic evacuation simulation greatly improved the efficiency of personnel evacuation, and the simulation results accord with actual situation. For disaster diffusion, repelling force in the simulation results, suggests there should be rational layout in public, and when designing the building, such as the evacuation passageway, we should consider the curve relationship between personnel repulsion and evacuation efficiency if in emergency. We should make the repulsive force in safety. For indoor installation of emergency facilities, we should try to reduce the harm of disaster. In a word, designing a set of real-time dynamic monitoring system, indoor reasonable planning system, rapid real-time deployment of dynamic evacuation plan for your particular situation, are the most effective ways in terms of protection and evacuees.

\section{Reference}

[1]Helbing D, Farkas I, Vicsek T. Simulating dynamical features of escape panic [J]. Nature, 2000, 407: 487-490.

[2]Shiraki W, Inomo H, Ishikawa H, Yasuda K, Aritomo H. Simulation of pedestrian dynamics at occurrence of disaster using CA-model [J]. Proceedings of the 2004 International Conference on Intelligent Mechatronics and Automation, 2004: 191-195.

[3]Haque ME, Balasubramanian S. Fire emergency building evacuation: A computer simulation model [J]. Computers and Their Applications, 2004: 70-74.

[4]Chunshan lv, Wenguo Weng, RuiYang and so on. Movement patterns and evacuation model under cellular automaton on fire [J]. Journal of Tsinghua university, 2007(12):2163-2167.

[5]Ying $\mathrm{Wu}$, Zhuoqiu Li. Evacuation model research for crowd on fire in the high building [J].Safety and Environment Engineering, 2008, 15(3):87-89.

[6]Yujin Zhang, Qinkun Xu, ShixiongLi. Numerical simulation of smoke and fire movement in dormitory corridor of university [J]. Journal of southwest university of science and technology, 2013, 28(1):49-53. 